\title{
Metabolic syndrome in older subjects: Coincidence or clustering?
}

\author{
Mario Bo*, Alessandro Sona, Marco Astengo, Umberto Fiandra, Eleonora Quagliotti, \\ Alessia Brescianini, Gianfranco Fonte
}

Section of Geriatrics, Department of Medical and Surgical Disciplines, University of Turin, Azienda Ospedaliera San Giovanni Battista, Molinette, Corso Bramante 88, I-10126 Torino, Italy

\section{A R T I C L E I N F O}

\section{Article history:}

Received 6 August 2007

Received in revised form 1 December 2007

Accepted 5 December 2007

Available online 19 February 2008

\section{Keywords:}

Metabolic syndrome

Elderly

Structural equation modeling

\begin{abstract}
A B S T R A C T
The prevalence of the metabolic syndrome (MS) increases with advancing age. However, aging per se is associated with increased prevalence of most of the abnormalities contributing to the MS. Whether MS in older people consistently identifies a true pathophysiological entity or a casual aggregation of agingassociated metabolic abnormalities, remains to be fully elucidated. In the present study, we aimed to evaluate whether in older subjects the aggregation of metabolic components of the MS, as defined by the National Cholesterol Education Program Adult Treatment Panel III (NCEP-ATP III), is consistent with a single latent variable. Age, waist circumference, systolic and diastolic blood pressure, metabolic variables were determined in 152 older ( $>70$ years), non-diabetic, healthy men. Cronbach alpha was used to assess the internal consistency of the components contributing to the MS. Structural equation modeling, using the Normed Fit Index (NFI), the Root Mean Square Error of Approximation (RMSEA), the Comparative Fit Index (CFI), and the Tucker-Lewis Index (TLI) was used to assess the fit to a model with a single latent variable. The Cronbach alpha test showed low internal consistency among the metabolic variables $(\alpha=0.31)$. The calculated $\chi^{2}$ values were 28.31 and 32.52 for model entering hypertension as dichotomous variable and for model entering blood pressure values, respectively, both expressing low fit to a model with a single latent variable. In both models, CFI (0.41 and 0.55), NFI (0.59 and 0.55), RMSEA $(0.25$ and 0.22$)$ and TLI $(-0.31$ and -0.12$)$ scores showed a low fit of the metabolic alterations to a single latent variable. These findings suggest caution in making diagnosis of MS at older ages, since metabolic and cardiovascular abnormalities being per se extremely common in elderly people, do not appear to cluster together under a single common factor.
\end{abstract}

(c) 2008 Published by Elsevier Ireland Ltd.

\section{Introduction}

The term MS denotes a condition recognized by the cooccurrence of multiple metabolic abnormalities, including central obesity, abnormal fasting glucose, dyslipidemia and hypertension (Reaven, 1988; WHO, 1999). The MS has been associated with the development of diabetes and increased risk of cardiovascular morbidity and mortality, even in older subjects (Lempiainen et al., 1999; Lakka et al., 2002; Wannamethee et al., 2005a; McNeill et al., 2006), but several studies demonstrated that MS does not provide incremental risk information above and beyond its individual components (Yarnell et al., 1998; Lawlor et al., 2004a; Iribarren et al., 2006; Sundstrom et al., 2006a). Moreover, there remains a fundamental issue concerning the clinical nature of the syndrome, that is, whether MS is a true pathophysiological condition or

\footnotetext{
* Corresponding author. Tel.: +39 0116335 006; fax: +39 0116961045 . E-mail address: mario.bo@unito.it (M. Bo).
}

merely a variegated cluster of risk factors produced by expert groups (Kahn et al., 2005; Reaven, 2005; Grundy, 2006a). The prevalence of MS is reported to rise dramatically with increasing age (Ford et al., 2002; Cameron et al., 2004). However, aging per se is associated with increased incidence and prevalence of most of the abnormalities contributing to the MS, including reduced lean/ fat mass ratio, hypertension, alterations in glucose homeostasis and decreased high density lipoprotein (HDL) concentration. While it appears plausible that at young-adult age a single pathophysiological alteration (increased visceral adipose tissue and/or insulin-resistance) might drive the early and unusual clustering of metabolic abnormalities (dyslipidemia, hypertension, abnormal glucose tolerance and inflammation) (Shen et al., 2003; Bo et al., 2004; Kahn et al., 2005; Reaven, 2005; Pladevall et al., 2006), it seems much more hazardous to cluster in a syndrome risk factors which are extremely common in older subjects. Therefore, whether MS in older people consistently identifies a true pathophysiological entity or a casual aggregation of agingassociated metabolic abnormalities remains to be fully elucidated. 
On this background, we aimed to evaluate whether in older non-diabetic subjects the aggregation of metabolic components contributing to the MS is consistent with a common single syndromic variable. Therefore, several statistical procedures were used to assess whether a model with a single latent variable may account for the variables contributing to MS in older subjects.

\section{Subjects and methods}

An invitation to participate to the study was sent to five general practitioners working in different districts of the metropolitan area in Turin. A detailed description of the study, including design, aims and inclusion/exclusion criteria, was provided. A descriptive letter of the study was attached for eligible patients, in order to obtain an informed consent to participation. Men aged 70 years or older were eligible to the study. Further inclusion criteria were independence in daily living, i.e., score $<1$ on the index of activities of daily living (ADL) (Katz et al., 1963) and absence of cognitive impairment, i.e., score $<4$, on the short portable mental status questionnaire (SPMSQ) (Pfeiffer, 1975). Exclusion criteria were: known diabetes, hepatic or renal failure, conditions or disorders affecting metabolism, use of lipid-lowering drugs in the previous month, neoplasm or other severe diseases. Eligible subjects were enrolled in the period September-October 2006.

A detailed medical history was collected and the following variables were considered: age, smoking habits, alcohol consumption, and cardiovascular history. In each patient the following measures were obtained: weight $(\mathrm{kg})$, height $(\mathrm{m})$, body mass index (BMI), calculated according to the formula weight $(\mathrm{kg}) /$ height $\left(\mathrm{m}^{2}\right)$, waist and hip circumferences $(\mathrm{cm})$ measured according to current guidelines (WHO, 1995), systolic and diastolic blood pressure (SBP and DBP, respectively) (mean value of 3 measures obtained with an appropriately sized cuff and standard mercury sphygmomanometer at the non-dominant upper arm after a 5-min rest in the supine position).

Blood samples were collected from an antecubital vein into vacutainer tubes containing EDTA after a 12-h overnight fast for routine blood chemistry, measurement of plasma lipid and lipoprotein levels, coagulative and inflammatory variables. Total cholesterol (TC) and triglycerides (TG) were measured using standard commercial enzymatic kits (CHOD-PAP and GPO-PAP methods, Roche Diagnostics, Mannheim, Germany). HDL-cholesterol (HDL-C) levels were measured through enzymatic colorimetric assay by a direct method (ADVIA 1650/2400, Bayer, Milano, Italy) after separation of cholesterol from non-HDL particles. LDL-cholesterol (LDL-C) concentration was calculated according to the formula of Friedewald et al. (1972). Plasma fibrinogen was quantified automatically through a functional coagulative assay according to the Clauss method (STA-Fibrinogen, Roche). Pentameric C-reactive protein (pCRP) levels were measured with a highly sensitive immunoassay that used a monoclonal antibody coated with polistirene particles; the assay was performed using a Behring BN-100 nephelometer (DADE Behring, Marburg, Germany), according to the method described by the manufacturer (Ledue et al., 1998; Roberts et al., 2001). Glucose was enzymatically determined by the hexokinase method. Serum insulin was determined by monoclonal antibody method (Insulin IRMA CT, RADIM, Pomezia, Italy). Insulinresistance was calculated through the homeostasis model assessment (HOMA) according to the formula: fasting serum insulin $(\mu \mathrm{U} / \mathrm{ml}) \times$ fasting plasma glucose $(\mathrm{mmol} / \mathrm{l}) / 22.5$ (Galvin et al., 1992; Bonora et al., 2000).

Diagnosis of MS was made according to the NCEP-ATP III guidelines (Expert Panel, 2001). Participants taking anti-hypertensive medications were counted as meeting the blood pressure criterion (He et al., 2006). Patients with a new finding of diabetes were not included in the study.

The study protocol agreed with the recommendations of the World Medical Association for biomedical research involving human subjects.

\subsection{Statistical analysis}

The sample size was defined according to the structural equation modeling. In the absence of a definite rule, we adopted one of the several arbitrary "rules of thumb" proposed: at least 10 cases for item according to Bryant and Yarnold (1995) and at least 100 cases overall according to Hatcher et al. (1995).

Data were analyzed using SPSS for Windows 12 and Tetrad 4.0. Frequencies, mean and standard deviation (SD), skewness and kurtosis were calculated. Non-normally distributed variables were normalized according to Blom (1959). The Cronbach alpha test (score range $0-1$ : a score of 1 defines the maximal internal consistency) was used to assess the internal consistency of the components contributing to MS. The structural equation modeling methodology was used to assess the fit to a model with a single latent variable. This was assessed using the NFI, the RMSEA, the CFI, and the TLI. These fit indexes were appropriately used to minimize the effect of the sample size on the $\chi^{2}$ values. The score range for NFI, CFI and TLI is 0-1: a score of 1 identifies the optimal fit, and score $\geq 0.9$ are usually considered expression of a good fit. For the TLI negative values are considered equal to 0 . For the RMSEA, negative values are conventionally considered equal to 0 ; values $<0.05$ suggest a good fit.

\section{Results}

A total of 156 subjects were selected for the study. Three of them received a new diagnosis of diabetes and were excluded from the study; one subject refused to participate. Analysis was conducted on 152 subjects.

Characteristics of the sample investigated are shown in Table 1. Mean age was $73.1 \pm 4.7$ years. SBP values $\geq 130 \mathrm{mmHg}$ and DBP values $\geq 85 \mathrm{mmHg}$ were found in $128(84.2 \%)$ and 98 (64.5\%) subjects, respectively; 94 subjects (61.8\%) had elevated SBP and DBP. Seventy subjects (46\%) were on anti-hypertensive medication. Mean waist circumference was $96.0 \pm 12.5 \mathrm{~cm}$ and 92 subjects (60.5\%) had central obesity according to the NCEP-ATP III criteria. Mean fasting

Table 1

Characteristics of the sample at baseline

\begin{tabular}{ll}
\hline Parameters & Values \\
\hline Age (years) & $73.1 \pm 4.7$ \\
SBP $(\mathrm{mmHg})$ & $142 \pm 17$ \\
DBP $(\mathrm{mmHg})$ & $87 \pm 8$ \\
Median BP $(\mathrm{mmHg})$ & $106 \pm 10$ \\
BMI $\left(\mathrm{kg} / \mathrm{m}^{2}\right)$ & $25.4 \pm 3.5$ \\
Waist circumference $(\mathrm{cm})$ & $96 \pm 12.5$ \\
Fasting glucose $(\mathrm{mg} / \mathrm{dl})$ & $90.9 \pm 13.3$ \\
Fasting insulin $(\mathrm{mU} / \mathrm{l})^{\mathrm{a}}$ & $16.5(13.2-19.8)$ \\
HOMA-IR & $3.73(2.7-5.0)$ \\
TC $(\mathrm{mg} / \mathrm{dl})^{\mathrm{a}}$ & $217.5(187.0-240.2)$ \\
HDL-C $(\mathrm{mg} / \mathrm{dl})$ & $64.3 \pm 15.2$ \\
LDL-C (mg/dl) & $128.2(106.5-150.3)$ \\
TG $(\mathrm{mg} / \mathrm{dl})^{\mathrm{a}}$ & $107.5(83.5-134.2)$ \\
Apo-B $(\mathrm{mg} / \mathrm{dl})^{\mathrm{a}}$ & $108(95.2-132.8)$ \\
CRP $(\mathrm{mg} / \mathrm{l})^{\mathrm{a}}$ & $1.85(0.8-3.8)$ \\
Fibrinogen $(\mathrm{mg} / \mathrm{dl})$ & $386 \pm 70$ \\
Erythrocyte sedimentation rate $(\mathrm{mm} / \mathrm{h})$ & $16.7 \pm 11.6$ \\
Creatinine $(\mathrm{mg} / \mathrm{dl})^{\mathrm{a}}$ & $0.81(0.75-0.95)$ \\
\hline
\end{tabular}

Mean \pm S.D., unless otherwise indicated.

a Median and interquartile range. 


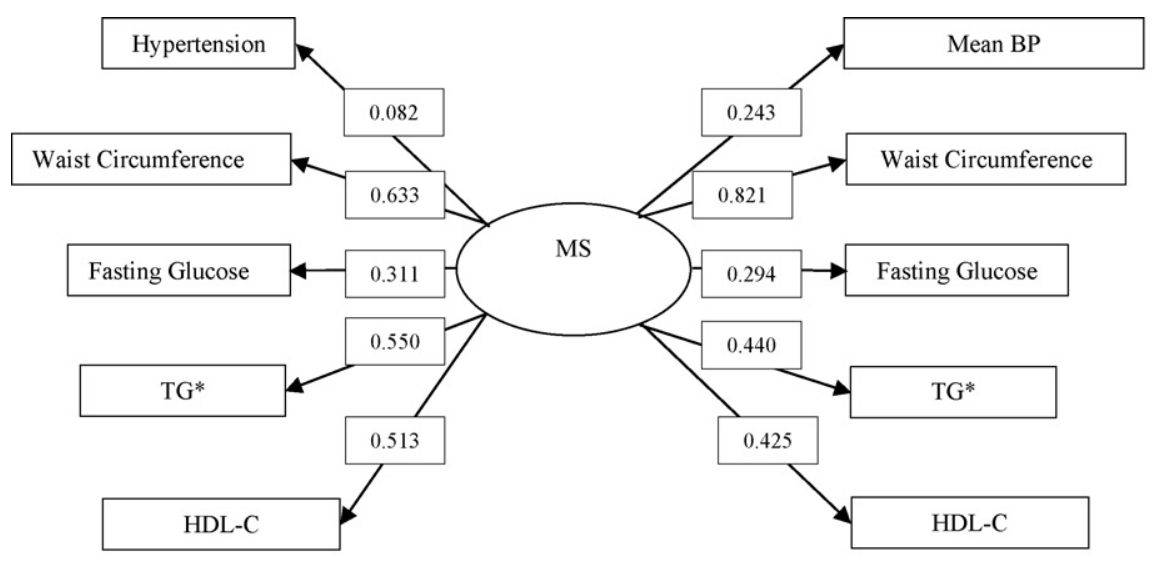

$$
\begin{aligned}
& \text { Degrees of freedom }=4 \\
& \chi^{2}=28.101 \\
& p<0.0001
\end{aligned}
$$

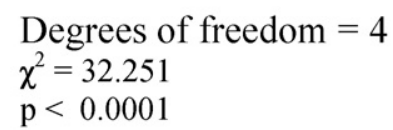

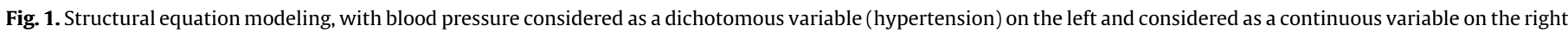
side of the scheme. *Triglyceridemia was normalized according to Blom (1959).

Table 2

Results of fit indices with BP considered as a dichotomous variable $(A)$ and considered as a continuous variable $(B)$

\begin{tabular}{lrr}
\hline Parameters & \multicolumn{2}{l}{$A$} \\
\hline CFI & 0.41 & 0.55 \\
RMSEA & 0.25 & 0.22 \\
NFI & 0.59 & 0.55 \\
TLI & -0.31 & -0.12 \\
\hline
\end{tabular}

glucose was $90.9 \pm 13.3 \mathrm{mg} / \mathrm{dl}$ and 40 subjects (26.3\%) had fasting glucose $>110 \mathrm{mg} / \mathrm{dl}$. Median TG value was $107.5 \mathrm{mg} / \mathrm{dl}$ and 26 subjects (17.1\%) had values exceeding $150 \mathrm{mg} / \mathrm{dl}$. Mean HDL-C was $64.3 \pm 15.2 \mathrm{mg} / \mathrm{dl}$; values lower than $40 \mathrm{mg} / \mathrm{dl}$ were observed in 6 subjects $(3.9 \%)$.

The presence of MS according to the NCEP-ATP III criteria was diagnosed in 40 subjects (26.3\%). The most common clusters accounting for diagnosis of MS were hypertension-impaired fasting glucose-central obesity (41\%), hypertension-central obesity-hypertriglyceridemia (19\%), hypertension-impaired fasting glucose-hypertriglyceridemia (12\%).

The Cronbach alpha test showed low internal consistency among the metabolic variables $(\alpha=0.31)$. Results of the structural equation modeling are shown in Fig. 1 and Table 2. The calculated $\chi^{2}$ were 28.31 and 32.52 for the model entering hypertension as dichotomous variable and for the model entering blood pressure values, respectively, both expressing low fit to a model with a single latent variable. In both models, CFI (0.41 and 0.55), NFI (0.59 and 0.55$)$, RMSEA ( 0.25 and 0.22 ) and TLI ( -0.31 and -0.12$)$ scores showed a low fit of the metabolic alterations to a single latent variable. As a whole, these results suggest that in this sample of older subjects, a single latent variable may not account for variables contributing to the definition of the MS.

\section{Discussion}

The results of the present study suggest that MS should not always be regarded as a coherent clinical entity in older subjects. In this sample of healthy older subjects, the structural equation modeling showed that the metabolic components act as indepen- dent variables. Moreover, fit model analysis (NFI, RMSEA, CFI and TLI) and Cronbach test demonstrated a poor internal consistency of the MS. As a whole, these findings suggest caution in making diagnosis of MS at older ages, since metabolic and cardiovascular abnormalities, which are per se extremely common in elderly people, do not appear to cluster together under a single common factor.

Several approaches have been used to confirm the existence of MS and to identify its pathophysiological mechanisms. At first, epidemiologic surveys demonstrated that the aggregation of some metabolic alterations was more frequent than would be expected by chance (Ramachandran et al., 1998; Wilson et al., 1999). Several studies used different factorial analyses to explore the underlying structure of the MS (Godsland et al., 1998; Gray et al., 1998; Levya et al., 1998; Chen et al., 1999; Kekalainen et al., 1999; Lempiainen et al., 1999). Lempiainen et al. (1999) showed that BMI, waist-to-hip ratio, TG, fasting plasma glucose and insulin clustered on a single factor in both sexes; in a similar study on younger non-diabetic subjects (Meigs et al., 1997), 3 distinct factors were found, the first of them including hyperinsulinemia, hyperglycemia, high levels of TG, low HDL-C, high BMI and high waist-to-hip ratio. A separate hypertension factor was reported by both authors. However, a common soil of the syndrome was not identified in most of these studies, and factorial analyses steadily showed the presence of three or more metabolic clusters within the syndrome. This is specifically the case of blood pressure values, which have been shown to aggregate separately from other metabolic clusters. Pladevall et al. (2006) used confirmatory factor analysis on three large patient datasets. They showed that insulin-resistance, mean arterial pressure, TG-to-HDL-ratio and waist circumference clustered together under a single latent factor, suggesting the existence of a common causal factor underlying these different components. However, they used an original approach introducing only one measure for each of the four postulated metabolic syndrome components, namely HOMA-IR for insulin-resistance, mean arterial pressure for SBP and DBP values, the ratio of TG-to-HDL-C for the dyslipidemia trait, and waist circumference for central obesity. In our view, such a pathophysiological approach profoundly differs from the NCEP-ATP III diagnostic criteria currently recommended to recognize the MS in the clinical practice. 
Each abnormality contributing to the MS is also a conventional cardiovascular risk factor and the coexistence of such alterations in a single person can be the result of two mechanisms: coincidence or clustering. It is no matter of discussion that aging is associated through several pathophysiological mechanisms with an increasing prevalence of risk factors and that, as a consequence, this frequent coexistence of multiple metabolic alterations makes it easier to reach the number of criteria necessary for the diagnosis of MS. However, coexistence does not imply clustering, the latter being supported either by statistical and biological links. As an example of this assumption, we can consider how the prevalence of MS in this sample of older subjects changed when substituting the International Diabetes Federation (IDF) criteria (Alberti et al., 2005 ) for the NCEP-ATP III criteria: $16.1 \%$ vs. $26.3 \%$. These two definitions are considered very congruent (Grundy, 2006b) because each contains five virtually identical components; however, they substantially differ in that the IDF definition requires the mandatory presence of central obesity. Central obesity was agreed as essential, because of the evidence linking waist circumference with cardiovascular disease and the other MS components, and the likelihood that central obesity is an early step in the etiological cascade leading to full MS. Therefore, although the criteria are identical, those endorsed by the IDF underscore the potential pathophysiological mechanisms more strongly than the simple aggregation of components in the NCEP-ATP III definition does.

Beyond the skepticism on the common biological basis for the diagnostic NCEP-ATP III algorithm, our findings in older subjects provide some evidence that there is low internal consistency among the metabolic variables and that a single latent factor might not account for variables contributing to the definition of the MS. There have been very few scientific evidence presented or discussed for why the ATP III algorithm is what it is and several concerns have been raised about the arbitrary cut-off selected for most of the metabolic components (Kahn, 2006). Moreover, these items have not been critically addressed in older subjects, who usually have the greatest burden of cardiovascular and metabolic risk factors. On this background, we decided to use a comprehensive approach to evaluate either the internal consistency of the syndrome (with the Cronbach alpha test) and the fit of the components to a single latent variable (through the structural equation modeling as confirmatory factor analysis) specifically in older subjects (Kline, 1998; Shen et al., 2003; Lawlor et al., 2004b). Structural equation modeling implies an "a priori" definition of the number of factors to be extracted; we decided to extract a single latent factor, i.e., the MS that we hypothesized to be a pathophysiological entity.

Most important, the present study was not addressed to investigate the consistency of the biological mechanisms underlying the MS, but just to evaluate the statistical coherence of it among older healthy subjects. Therefore, biological inferences should not be made on the basis of these findings.

Some limitations of the present study must be addressed. Despite the limited sample size, the prevalence of MS observed in our study was keeping with results of previous studies (Lawlor et al., 2004a; He et al., 2006; McNeill et al., 2006; Ravaglia et al., 2006; Sundstrom et al., 2006b) and slightly higher than that reported in recent national surveys (Wannamethee et al., 2005b, 2006; Cankurtaran et al., 2006; Maggio et al., 2006). Only older men were enrolled in this study, which obviously limits the generalization of these findings. There was a high prevalence of subjects with blood pressure values above the NCEP-ATP III systolic and diastolic cut-off values. However, most of these subjects were currently receiving blood pressure lowering drugs. Patients were considered hypertensive either when treated with anti-hyperten- sive drugs or when they had blood pressure values exceeding the cut-off values without anti-hypertensive treatment. Analysis of the blood pressure variable was performed either introducing dichotomous and continuous variables. Further concerns about the possibility to extend our observations to a general population of aged subjects could be raised because of the very low prevalence of hypo-HDL-C. This can be largely explained by our decision to enroll healthy independent elderly, who, on the other hand, would be the main target of geriatric cardiovascular prevention.

There remain several uncertainties about the utility in diagnosing MS in older subjects. Several studies (Yarnell et al., 1998; Lawlor et al., 2004b; Wilson et al., 2005; Sundstrom et al., 2006a,b) showed that the risk of cardiovascular diseases associated with the syndrome is not greater than that explained by the presence of its single components. Other studies demonstrated that MS does not consistently predict adverse events and mortality in elderly patients (McNeill et al., 2006; Ravaglia et al., 2006; Sundstrom et al., 2006b). However, the basic question is whether we know enough about this constellation of risk factors to warrant adopting a unique clinical construct that has value to either physicians or patients. On this point, our findings demonstrate that in a sample of healthy older subjects there is low internal consistency among the metabolic variables and that there is a low fit of these variables to a single latent model. These findings should suggest caution in labeling as MS any aggregation of metabolic abnormalities according to the NCEP-ATP III "three out of five" rule (Dominiczak, 2006); some of these patients will have MS, some of them will suffer from multiple risk factors. In light of persisting uncertainties about predictive value of MS above that of its single components in this age group, physician should be addressed to recognize and treat metabolic abnormalities either they are part of MS or not.

\section{References}

Alberti, K.G.M.M., Zimmet, P., Shaw, J., 2005. For the IDF Epidemiology Task Force Consensus Group. The metabolic syndrome-a new worldwide definition. Lancet 366, 1059-1062.

Blom, G., 1959. Statistical Estimates and Transformed Beta Variables. Wiley and Sons, New York

Bo, M., Raspo, S., Morra, F., Isaia, G., Cassader, M., Fabris, F., Poli, L., 2004. Body fat and C-reactive protein levels in healthy non-obese men. Nutr. Metab. Cardiovasc. Dis. $14,66-72$.

Bonora, E., Targher, G., Alberiche, M., Bonadonna, R.C., Saggiani, F., Zenere, M.B., Monauni, T., Muggeo, M., 2000. Homeostasis model assessment closely mirrors the glucose clamp technique in the assessment of insulin sensitivity. Diabetes Care 23, 57-63.

Bryant, F.B., Yarnold, P.R., 1995. Comparing five alternative factor-models of the Student Jenkins Activity Survey: separating the wheat from the chaff. J. Pers. Assess. 64, 145-158.

Cameron, A.J., Shaw, J.E., Zimmet, P.Z., 2004. The metabolic syndrome: prevalence in worldwide populations. Endrocrinol. Metab. Clin. North Am. 32, 351-375.

Cankurtaran, M., Halil, M., Yavuz, B.B., Dagli, N., Oyan, B., Ariogul, S., 2006. Prevalence and correlates of metabolic syndrome (MS) in older adults. Arch. Gerontol. Geriatr. 42, 35-45.

Chen, W., Srinivasan, S.R., Elkasabany, A., Berenson, G.S., 1999. Cardiovascular risk factors clustering features of insulin resistance syndrome (syndrome $\mathrm{X}$ ) in a biracial (black-white) population of children, adolescents, and young adults: The Bogalusa Heart Study. Am. J. Epidemiol. 150, 667-674.

Dominiczak, M.H., 2006. Atherosclerosis: metabolic syndrome-a science or not a science? Curr. Opin. Lipid. 17, 609-611.

Expert Panel on Detection, Evaluation and Treatment of High Blood Cholesterol in Adults, 2001. Executive Summary of the Third Report of the National Cholesterol Education Program (NCEP) Expert Panel on Detection, Evaluation and Treatment of High Blood Cholesterol in Adults (Adult Treatment Panel III). J. Am. Med. Assoc. 285, 2486-2497.

Ford, E.S., Giles, W.H., Dietz, W.H., 2002. Prevalence of the metabolic syndrome among US adults: findings from the third National Health and Nutritional Examination Survey. J. Am. Med. Assoc. 287, 356-359.

Friedewald, W.T., Levy, R.I., Fredrickson, D.S., 1972. Estimation of the concentration of low-density lipoprotein cholesterol in plasma without the use of the preparative ultracentrifuge. Clin. Chem. 18, 499-502.

Galvin, P., Ward, G., Walters, J., Pestell, R., Koschmann, M., Vaag, A., Martin, I., Best, J.D., Alford, F., 1992. A simple method for quantitation of insulin sensitivity 
and insulin release from an intravenous glucose tolerance test. Diabet. Med. 9, 921-928.

Godsland, I.F., Levya, F., Walton, C., Worthington, M., Stevenson, J.C., 1998. Associations of smoking, alcohol and physical activity with risk factors for coronary heart disease and diabetes in the first follow-up cohort of the Heart Disease and Diabetes Risk Indicators in a Screened Cohort study (HDDRISC-1). J. Int. Med. 244, 33-41

Gray, R.S., Fabsitz, R.R., Cowan, L.D., Lee, E.T., Howard, B.V., Savage, P.J., 1998. Risk factor clustering in the insulin resistance syndrome: The Strong Heart Study. Am. J. Epidemiol. 148, 869-878.

Grundy, S.M., 2006a. Does the metabolic syndrome exist? Diabetes Care 29, 16891692.

Grundy, S.M., 2006b. Metabolic syndrome: connecting and reconciling cardiovascular and diabetes world. J. Am. Coll. Cardiol. 47, 1093-1100.

Hatcher, R.L., Barends, A., Hansell, J., Gutfreund, M.J., 1995. Patients' and therapists' shared and unique views of the therapeutic alliance: an investigation using confirmatory factor analysis in a nested design. J. Consult. Clin. Psychol. 63. 636-643.

He, Y., Jiang, B., Wang, J., Feng, K., Chang, Q., Fan, L., Li, X., Hu, F.B., 2006. Prevalence of the metabolic syndrome and its relation to cardiovascular disease in an elderly Chinese population. J. Am. Coll. Cardiol. 47, 1588-1594.

Iribarren, C., Go, A.S., Husson, G., Sidney, S., Fair, J.M., Quertermous, T., Hlatky, M.A., Fortmann, S.P., 2006. Metabolic syndrome and early-onset coronary artery disease. Is the whole greater than its parts? J. Am. Coll. Cardiol. 48, 1800-1807.

Kahn, R., 2006. The metabolic syndrome (emperor) wears no clothes. Diabetes Care 29, 1693-1696.

Kahn, R., Buse, J., Ferrannini, E., Stern, M., 2005. The metabolic syndrome: time for a critical appraisal. Diabetes Care 28, 2289-2304.

Katz, S., Ford, A.B., Moskowitz, R.W., Jackson, B.A., Jaffe, M.W., 1963. Studies of illness in the aged. The index of ADL, a standardized measure of biological and psychosocial function. J. Am. Med. Assoc. 185, 914-919.

Kekalainen, P., Sarlund, H., Pyorala, K., Laakso, M., 1999. Hyperinsulinemia cluster predicts the development of type 2 diabetes independent of a family history of diabetes. Diabetes Care 22, 86-92.

Kline, R.B., 1998. Principles and Practice of Structural Equation Modeling. Guilford Press, New York.

Lakka, H.M., Laaksonen, D.E., Lakka, T.A., Niskanen, L.K., Kumpusalo, E., Tuomilehto, J., Salonen, J.T., 2002. The metabolic syndrome and total and cardiovascular disease mortality in middle-aged men. J. Am. Med. Assoc. 288, 2709-2716.

Lawlor, D.A., Ebrahim, S., Davey Smith, G., 2004a. The metabolic syndrome and coronary heart disease in older women: findings from the British Women's Heart and Health Study. Diabet. Med. 21, 906-913.

Lawlor, D.A., Ebrahim, S., May, M., Davey Smith, G., 2004b. (Mis)use of factor analysis in the study of insulin resistance syndrome. Am. J. Epidemiol. 159, 1013-1018.

Ledue, T.B., Weiner, D.L., Sipe, J.D., Poulin, S.E., Collins, M.F., Rifai, N., 1998. Analytical evaluation of particle-enhanced immunonephelometric assays for C-reactive protein, serum amyloid $A$ and mannose-binding protein in human serum. Ann. Clin. Biochem. 35, 745-753.

Lempiainen, P., Mykkanen, L., Pyorala, K., Laakso, M., Kuusisto, J., 1999. Insulin resistance syndrome predicts coronary heart disease events in elderly nondiabetic men. Circulation 100, 123-128.

Levya, F., Godsland, I.F., Worthington, M., Walton, C., Stevenson, J.C., 1998. Factors of the metabolic syndrome: baseline interrelationships in the first follow-up cohort of the HDDRISC Study (HDDRISC-1). Arterioscler. Thromb. Vasc. Biol. 18, 208-214.

Maggio, M., Lauretani, F., Ceda, G.P., Bandinelli, S., Basarla, S., Ble, A., Egan, J., Prolisso G., Najjar, S., Metter, E.J., Valenti, G., Guralnik, J.M., Ferrucci, L., 2006. Association between hormones and metabolic syndrome in older Italian men. J. Am. Geriatr. Soc. $54,1832-1838$.
McNeill, A.M., Katz, R., Girman, C.J., Rosamond, W.D., Wagenknecht, L.E., Barzilay J.I., Tracy, R.P., Savage, P.J., Jackson, S.A., 2006. Metabolic syndrome and cardiovascular disease in older people: the Cardiovascular Health Study. J. Am. Geriatr. Soc. 54, 1317-1324.

Meigs, J.B., D’Agostino, R.B., Wilson, P.W.F., Cupples, L.A., Nathan, D.M., Singer, D.E., 1997. Risk variable clustering in the insulin resistance syndrome. The Framingham offspring study. Diabetes 46, 1594-1600.

Pfeiffer, E., 1975. A short portable mental status questionnaire for the assessment of organic brain deficit in elderly patients. J. Am. Geriatr. Soc. 23, 433-441.

Pladevall, M., Singal, B., Williams, L.K., Brotons, C., Guyer, H., Saturni, J., Falces, C., Serrano-Rios, M., Gabriel, R., Shaw, J.E., Zimmet, P.Z., Haffner, S., 2006. A single factor underlies the metabolic syndrome. A confirmatory factor analysis. Diabetes Care 29, 113-122.

Ramachandran, A., Snchalatha, C., Latha, E., Satyavani, K., Vijay, V., 1998. Clustering of cardiovascular risk factors in urban Asian Indians. Diabetes Care 21, 967-971.

Ravaglia, G., Forti, P., Maioli, F., Bastagli, L., Chiappelli, M., Montesi, F., Bolondi, L. Patterson, C., 2006. Metabolic syndrome. Prevalence and prediction of mortality in elderly individuals. Diabetes Care 29, 2471-2476.

Reaven, G.M., 1988. Role of insulin resistance in human disease. Diabetes 37, 1595 1607.

Reaven, G.M., 2005. The metabolic syndrome: requiescat in pace. Clin. Chem. 51, 931-938.

Roberts, W.L., Moulton, L., Law, T.C., Farrow, G., Cooper-Anderson, M., Savory, J., Rifai, N., 2001. Evaluation of nine automated high-sensitivity C-reactive protein methods: implications for clinical and epidemiological applications. Part 2. Clin. Chem. 47, 418-425.

Shen, BJ., Todaro, J.F., Niaura, R. McCaffery, J.M., Zhang J., Spiro, A., Ward, K.D. 2003. Are metabolic risk factors one unified syndrome? Modeling the structure of the metabolic syndrome X. Am. J. Epidemiol. 157, 701-711.

Sundstrom, J., Vallhagen, E., Riserus, U., Byberg, L., Zethelius, B., Berne, C., Lind, L., Ingelsson, E, 2006a. Risk associated with the metabolic syndrome versus the sum of its individual components. Diabetes Care 29, 1673-1674.

Sundstrom, J., Riserus, U., Byberg, L., Zethelius, B., Lithell, H., Lind, L., 2006b. Clinical value of the metabolic syndrome for long term prediction of total and cardiovascular mortality: prospective, population based cohort study. Br. Med. J. 332, $878-882$.

Wannamethee, S.G., Shaper, A.G., Lennon, L., Morris, R.W., 2005a. Metabolic syndrome vs. Framingham risk score for prediction of coronary heart disease, stroke and type 2 diabetes mellitus. Arch. Int. Med. 165, 2644-2650.

Wannamethee, S.G., Lowe, G.D.O., Shaper, A.G., Rumley, A., Lennon, L., Whincup P.H., 2005b. The metabolic syndrome and insulin resistance: relationship to haemostatic and inflammatory markers in older non diabetic men. Atherosclerosis $181,101-108$.

Wannamethee, S.G., Shaper, A.G., Whincup, P.H., 2006. Modifiable lifestyle factors and the metabolic syndrome in older men: affects of lifestyle changes. J. Am. Geriatr. Soc. 54, 1909-1914.

World Health Organization, 1995. Physical Status: the Use and the Interpretation of Anthropometry. WHO, Geneva.

World Health Organization, 1999. Definition, Diagnosis and Classi-fication of Diabetes Mellitus and its Complications. Report of a WHO consultation. Part 1. WHO, Geneva.

Wilson, P.W., Kannel, W.B., Silbershatz, H., D'Agostino, R.B., 1999. Clustering of metabolic factors and coronary heart disease. Arch. Int. Med. 159, 1104-1109.

Wilson, P.W., D’Agostino, R.B., Parise, H., Sullivan, L., Meigs, J.B., 2005. Metabolic syndrome as a precursor of cardiovascular disease and type 2 diabetes mellitus. Circulation 112, 3066-3072.

Yarnell, J.W.G., Patterson, C.C., Bainton, D., Sweetnam, P.M., 1998. Is meta-bolic syndrome a discrete entity in the general population? evidence from the Caerphilly and Speedwell population studies. Heart 79, 248-252. 